\title{
EVALUATION OF FASTING CAPLLLARY GLUCOSE AND FASTING PLASMA GLUCOSE AS SCREENING TESTS FOR DIABETES AND PREDIABETES AMONG ADULTS IN A SEMI-URBAN AREA IN THE KATHMANDU DISTRICT, NEPAL
}

\author{
Silvanus $V^{1}$, Kafle PP1, Pokhrel $A^{2}$, Baral BK', Pokhrel BR
}

${ }^{1}$ Department of Community Medicine, Department of Biochemistry, Nepal Medical College Teaching Hospital,

Attarkhel, Gokarneshwor-8, Kathmandu, Nepal

\begin{abstract}
The natural history of type 2 diabetes includes a preceding period of impaired fasting glucose or impaired glucose tolerance which is referred to as prediabetes. During the asymptomatic phase of prediabetes, an estimated $20-30 \%$ of persons may develop complications like retinopathy, cardiovascular disease, neuropathy and nephropathy. Screening and lifestyle management may help to delay or arrest progression to diabetes. In primary care settings, point of care devices that measure glucose in capillary blood can be used for diagnosis of diabetes. This study was carried out to evaluate the performance of fasting capillary glucose (FCG) and fasting plasma glucose (FPG) measurement in screening for diabetes and prediabetes among adults in a semi-urban community in the Kathmandu district of Nepal. An observational, cross-sectional study design was used and FCG, FPG performance was evaluated by the 2-hour plasma glucose levels (2-hr PG) following Oral glucose tolerance test (75g glucose) using WHO 1998 criteria. Linear regression was performed to assess correlation coefficient (r) between FPG, FCG and 2 hr PG. Bland Altman plot and Receiver operator characteristic (ROC) curves were constructed to assess concordance, measure ROC AUC and determine sensitivity and specificity of the measurements at recommended cut-off values for identifying diabetes and prediabetes. Among the study participants $(\mathrm{n}=162), 104$ were female and 58 were male. Prevalence of undiagnosed diabetes and prediabetes was $4.32 \%$ (95\% CI $1.75 \%$ to $8.70 \%$ ) and $7.14 \%$ (95\% CI 3.89\% to $12.58 \%$ ). Strong positive correlation was seen between FPG and FCG (Spearman's r 0.67). FPG \& FCG had a moderate positive correlation $(r=0.49 \& 0.45)$ with $2 \mathrm{hr}$ PG levels $(\mathrm{p}<0.0001)$. FCG and FPG ROC AUC was 0.91 ( $95 \%$ CI 0.85 to 0.97 ) and 0.87 (95\% CI 0.78 to 0.97 ) in comparison to 0.98 (95\% CI 0.97 to 1.0) for the gold standard $2 \mathrm{hr}$ PG. At $110 \mathrm{mg} / \mathrm{dl}$ and above, FCG had an optimal sensitivity and specificity of $84.21 \%$ and $81.12 \%$ in comparison to $47.37 \%$ and $100 \%$ for FPG. At $100 \mathrm{mg} / \mathrm{dl}$, the sensitivity and specificity of FCG was $100 \%$ and $51.75 \%$ in comparison to $57.89 \%$ and $97.20 \%$ for FPG. In conclusion, at each recommended cut-off value, FCG was more sensitive than FPG with no significant difference between ROC AUCs of the two tests. Hence, FCG may be a suitable, sensitive, and convenient screening tool for diabetes and prediabetes in community-based settings. Larger prospective studies may validate the cost-effectiveness and efficiency of similar screening strategies in the Nepalese community
\end{abstract}

\section{KEYWORDS}

Screening, diabetes, prediabetes, FCG, FPG, OGTT

\section{CORRESPONDING AUTHOR}

Dr. Vinutha Silvanus

Department of Community Medicine,

Nepal Medical College Teaching Hospital,

Attarkhel, Gokarneshwor-8, Kathmandu, Nepal

Email: silvanus.v@gmail.com

ORCID ID: 0000-0002-9559-001X

DOI: https://doi.org10.3126/nmcj.v21i4.27615 


\section{INTRODUCTION}

Type 2 diabetes (T2D) is a chronic metabolic disorder with raised blood glucose levels due to a defect in the secretion of insulin and insulin resistance. The natural history of T2D includes a preceding period of impaired glucose tolerance (IGT) or impaired fasting glucose (IFG). This intermediate stage between normal blood glucose levels and diabetes is referred to as prediabetes. ${ }^{1}$ They represent persons who are at risk of developing diabetes in the future. Annual estimates suggest that about 5 to $12 \%$ of persons with impaired glucose regulation have progressed to T2D. Due to the gradual asymptomatic onset, T2D may remain undiagnosed for years. ${ }^{2}$

Population based data among adults in high income countries such as Australia and USA suggested that the onset of diabetes may precede clinical diagnosis by 9 to 12 years. $^{3}$ During the asymptomatic phase of prediabetes an estimated $20-30 \%$ of persons may develop complications like retinopathy, cardiovascular disease, neuropathy and nephropathy. Screening for prediabetes along with lifestyle management may help to delay or arrest progression to diabetes. ${ }^{4}$ Global data on persons with diabetes (PWDs) suggests that about a half remain undiagnosed. An overwhelming $80 \%$ of the PWDs are believed to live in low and middle income countries (LMICs). ${ }^{5}$

Measurement of blood glucose levels is crucial in the early detection of diabetes and prediabetes. Glucose can be measured as venous plasma or capillary whole blood. Diagnostic criteria have provided equivalence estimates for the two methods. The World Health Organization (WHO), International Diabetes Federation (IDF) and American Diabetes Association (ADA) have recommended that the fasting plasma glucose (FPG), a 2-hour plasma glucose (2 hr PG) after oral glucose tolerance test (OGTT) using $75 \mathrm{~g}$ glucose and glycated haemoglobin (HbA1c) are appropriate diagnostic tests for diabetes. While the cut-offs for detecting diabetes is similar across the guidelines, the ADA has lowered the cut-off for IFG from $110 \mathrm{mg} / \mathrm{dl}$ to $100 \mathrm{mg} / \mathrm{dl}$ for the American population. ${ }^{6,7}$

The same diagnostic tests have been used to screen and identify persons with undiagnosed diabetes and prediabetes. In comparison with the recommended FPG and HbA1c cut-off values, the $2 \mathrm{hr}$ PG value has been found to identify more persons with diabetes. The concordance between FPG, 2hr PG and HbA1c measurements is not perfect and these tests may not detect diabetes in the same individual. ${ }^{7}$
The 2016 WHO global report on diabetes suggested that among the LMICs, only 1 in 3 have access to basic technologies which can help to diagnose and manage persons with diabetes. In primary care settings where laboratory analysis of venous plasma glucose is difficult, point of care devices that measure glucose in capillary blood and meet ISO standards can be used for diagnosis of diabetes. ${ }^{8}$

The choice of screening tests varies with the resource settings. FPG, OGTT and are more commonly used in high resource settings. ${ }^{9}$ In the community setting, fasting capillary glucose (FCG), random capillary blood glucose (RCBG) and urine glucose testing may provide a good compromise between accuracy and cost effectiveness. ${ }^{10}$

Blood glucose meters are easy to use and inexpensive. However, they have a limited analytical measurement and can be inaccurate at the low and high blood glucose range.

The International Organization for Standardization (ISO:15197:2013) recommends that $95 \%$ of glucose meter results should be within $\pm 15 \mathrm{mg} / \mathrm{dl}$ when laboratory glucose values are $<100 \mathrm{mg} / \mathrm{dl}$; and the acceptable error should be within $\pm 20 \%$ for laboratory values $\geq 100 \mathrm{mg} /$ dl. However, ADA guidelines recommend an analytical error of $\leq 5 \%$ for all values. ${ }^{11,12}$

An assessment of the actual performance of 'approved glucose meters' in real life settings showed that a sizable proportion do not yield the specified readings. ${ }^{13}$

A systematic review has reported an increasing burden of diabetes in Nepal an LMIC, with a pooled prevalence of $8.4 \%$ among urban and rural populations. A higher prevalence of diabetes in urban populations may necessitate strategies for early screening. ${ }^{14}$

The Indian Diabetes Risk Score (IDRS) was developed as a simple, valid and easy to administer screening tool to detect diabetes and prediabetes among the South Indian population. It is comprised of four scored questions regarding age, abdominal obesity as measured by the waist circumference, family history of diabetes and physical activity levels. ${ }^{15}$

During a study that was planned to assess the performance of the IDRS as a screening tool among an adult population in a semi-urban setting in the Kathmandu district, Nepal, ${ }^{16}$ the performance of FCG was evaluated in comparison with FPG measurement in screening for diabetes and prediabetes. The concordance between FCG, FPG and $2 \mathrm{hr}$ PG measurements was also assessed. 


\section{MATERIALS AND METHODS}

A community-based cross-sectional, observational and analytical study was carried out in the Budhanilkantha municipality in Kathmandu District from September 2017 to May 2018.

Assuming a baseline prevalence of diabetes in Nepal of $8.4 \%$ as an equivalent proportion of undiagnosed diabetes, a $5.0 \%$ margin of error and a non-response rate of $20.0 \%$, the minimum sample size was calculated to be 142 adults who were not previously diagnosed with diabetes.

For evaluating the performance of FCG and FPG, all of the study participants were invited for an OGTT using 75g of glucose (WHO 1998 criteria) for definitive testing.

Sampling technique: Using a simple random sampling technique, 260 households were selected for a family survey from an estimated 600 households in the study area. Data collection was carried out in a step wise manner in order to increase the response rate for screening for diabetes and acquire the number needed to undertake definitive testing. ${ }^{17}$

Step 1: Adults over 20 years of age who were present in the household and not pregnant at the time of the survey were interviewed using a pretested structured questionnaire (modified STEPS survey) and IDRS screening tool after obtaining due written consent by trained UG medical students supervised by faculty from the department of Community Medicine..$^{18}$

Step 2: Physical measurement of height, weight, waist circumference and hip circumference was carried out at the household level as a part of the family study assessment.

Step 3: All of the study participants were invited to undergo an OGTT using $75 \mathrm{~g}$ of glucose as recommended by the WHO guidelines. ${ }^{1}$

Step 4: Estimation of the fasting capillary glucose (FCG) levels was offered to all the participants using a glucometer during a screening camp organized over 7 days in the community. Faculty from the departments of Community Medicine and Biochemistry conducted the screening camp along with the co-operation of community leaders and representatives, screened households and UG medical students. The volunteers were advised to consume the last meal by $8 \mathrm{pm}$ so that they were fasting for a minimum of 10 hours at the time of the testing. FPG was collected after the FCG estimation and $2 \mathrm{hr}$ PG samples were collected after the OGTT. Two similar glucometers were used throughout the screening period.
The camp began at 6:45 am in the morning at a predetermined venue in the community considering the convenience of the volunteers. FCG and fasting blood samples were collected by 8 a.m. and all the 2-hour OGTT samples were collected by 10 a.m. The volunteers were advised to rest and refrain from strenuous activities during the testing period and to report any adverse effects following glucose administration.

Principle of FCG estimation with glucometer: The VivaChek Ino Blood Glucose Monitoring System is designed to quantitatively measure the glucose concentration in fresh capillary whole blood. The glucose measurement is achieved by using the amperometric detection method. The test is based on measurement of electrical current caused by the reaction of the glucose with the reagents on the electrode of the test strip.

The blood sample is pulled into the tip of the test strip through capillary action. Glucose in the sample reacts with glucose enzyme and the mediator. Electrons are generated, producing a current that is correlated to the glucose concentration in the sample. After the reaction time, the glucose concentration in the sample is displayed. The meter is calibrated to display plasma-like concentration results.

Oral Glucose Tolerance Test: A fasting sample of venous blood was collected in a fluoride containing tube. The volunteers were given 75 grams of glucose dissolved in water (about 250$300 \mathrm{ml}$ ). The time of oral glucose administration was noted. Another venous blood sample was collected in a fluoride containing tube after 2 hours of glucose loading.

Collected venous blood samples were stored in a lab specimen transport bag and kept protected from direct sunlight. All specimens collected were delivered to the laboratory within 4 hours of collection for the separation of plasma from the cells. The samples were centrifuged at $3000 \mathrm{rpm}$ for 15 minutes and were estimated for plasma glucose by autoanalyzer (Johnson \& Johnson Vitros 250, USA) in using glucose oxidase method in the NMCTH laboratory.

Using the WHO 1999 consulting group criteria, diabetes was defined as FPG $\geq 126 \mathrm{mg} / \mathrm{dl}$ or $2 \mathrm{hr}$ PG $\geq 200 \mathrm{mg} / \mathrm{dl}$. Prediabetes was defined as two categories, IFG as FPG $\geq 110$ and $<126 \mathrm{mg} / \mathrm{dl}$ with $2 \mathrm{hr}$ PG $<140 \mathrm{mg} / \mathrm{dl}$. IGT, the second category in prediabetes was defined as FPG $<126 \mathrm{mg} / \mathrm{dl}$ and $2 \mathrm{hr}$ PG $\geq 140$ and $<200 \mathrm{mg} / \mathrm{dl}$. Prediabetes and diabetes were collectively labelled as 'Raised blood glucose'. Normoglycemia was defined as $\mathrm{FPG}<110 \mathrm{mg} / \mathrm{dl}$ and $2 \mathrm{hr} \mathrm{PG}<140 \mathrm{mg} / \mathrm{dl}{ }^{19,1}$ 
Persons with previously diagnosed diabetes during the screening period were labelled as 'known diabetes'.

A total of 306 persons were screened during the study period. Fifty persons were found to be previously diagnosed cases of diabetes. The study participants without diabetes $(n=256)$ were invited to undergo an OGTT. One hundred and sixty-two participants (63.28\%) responded to our invitation. One hundred and fiftynine participants underwent the OGTT. Three participants were tested for plasma glucose levels after a meal (post prandial) as they either vomited or were unable to drink the glucose solution. ${ }^{16}$

Data Analysis: Collected data was entered into forms designed in EPI-INFO version 7.2 and data was analysed using Stata 15IC licensed software.

The prevalence rate of undiagnosed diabetes and prediabetes (raised blood glucose) was reported with 95\% confidence intervals. Association between categorical data and gender was analysed using the Chi-square test. FCG, FPG, 2hr PG were reported as the median with interquartile range and graphically represented by boxplot grouped by gender. Normally distributed quantitative variables were reported as means with standard deviation.

To evaluate the performance of the screening tests, linear regression analysis was carried out between FPG and FCG measurements and between FPG and $2 \mathrm{hr}$ PG; and between FCG and $2 \mathrm{hr}$ PG to obtain the correlation co-efficient (r) and coefficient of determination $\left(\mathrm{r}^{2}\right)$. As the distribution of measurements was non-normal, the Spearman r value was reported.

Further, a Bland Altman (BA) plot was constructed as a visual tool to assess concordance between FCG and FPG measurements. The mean value of FPG and FCG measurements were plotted on the $\mathrm{x}$-axis and difference between the two measurements was plotted on the y-axis. As the distribution was non-normal, the median of all differences was plotted as a solid horizontal line and two additional dotted horizontal lines were plotted at the 2.5 and 97.5 percentile of the differences to indicate the limits of agreement. The median line indicated the systematic deviation between the two measurements. If $95 \%$ of the differences lie between the limits of the agreement, concordance between the measurements was assumed. ${ }^{20}$

FCG and plasma glucose concentration levels: Receiver operator characteristic (ROC) curves were constructed to determine the sensitivity and specificity of FCG and FPG at the recommended cut-off values for identifying diabetes and prediabetes. The area under the ROC curve (ROC
AUC) for FCG and FPG was reported with 95\% CI in comparison to the gold standard 2-hour PG. A non-parametric test was used to compare the equality of the FCG and FPG ROC AUC.

Ethical clearance for the study was obtained from the Nepal Medical College Institutional Review Committee.

\section{RESULTS}

Among the study participants ( $n=162), 104$ were female and 58 were male. The baseline characteristics categorized by gender were shown in Table 1. Educational status, occupation, abdominal obesity, alcohol use and smoking status were found to be significantly associated with gender $(p<0.05)$. More women had no formal schooling as compared to men. Alcohol use and smoking rate was higher among men.

The mean age and waist circumference were significantly higher among the men, though abdominal obesity was more prevalent among the women $(p<0.05)$, (Table $1 \& 2)$. There was no significant difference in the median levels of FCG, FPG and $2 \mathrm{hr}$ PG amongst the sexes, (Table 3), (Fig. 1).

The prevalence of undiagnosed diabetes was $4.32 \%$ (95\% CI $1.75 \%$ to $8.70 \%$ ) and that of prediabetes was $7.14 \%$ (95\% CI $3.89 \%$ to $12.58 \%$ ). The overall prevalence of persons with 'raised blood glucose' was $11.73 \%$ (95\% CI $5.64 \%$ to $21.28 \%$ ). All of the persons with prediabetes $(n=12)$ had IGT. Among them, three persons also had FPG between 110 to $125 \mathrm{mg} / \mathrm{dl}$. No person with isolated IFG was found. There was no significant association between gender and glycaemic status (Table 4).

The BA plot depicted a median difference of 24 $\mathrm{mg} / \mathrm{dl}$ between FPG and FCG measurements. The limits of agreement were 17 units above and 20.95 units below the median (Fig. 2).

Linear regression analyses between the glycaemic measurements was shown in Table 5. A strong positive correlation was seen between FPG and FCG with a Spearman's correlation coefficient (r) of 0.67 . Both of the measurements had a moderate positive correlation with the $2 \mathrm{hr}$ PG levels ( $\mathrm{r}=$ 0.49 and 0.45 respectively) $(\mathrm{p}<0.0001)$.

The FCG ROC AUC was 0.91 (95\% CI 0.85 to 0.97 ) as compared to 0.87 (95\% CI 0.78 to 0.97 ) for FPG and 0.98 (95\% CI 0.97 to 1.0 ) for the gold standard 2 hr PG for the identification of persons with raised blood glucose. There was no significant difference between the ROC AUC of FCG and FPG ( $p=0.40)$, (Fig. 3), (Table 6). 


\begin{tabular}{|c|c|c|c|c|c|}
\hline Variable & $\begin{array}{l}\text { Female } \\
(n=104)\end{array}$ & $\begin{array}{c}\text { Male } \\
(n=58)\end{array}$ & $\begin{array}{l}\text { Overall } \\
(n=162)\end{array}$ & $\begin{array}{l}\text { Chi square } \\
\text { value }\end{array}$ & $\begin{array}{c}\mathbf{P} \\
\text { value }\end{array}$ \\
\hline \multicolumn{6}{|l|}{ Age classification } \\
\hline $35-49$ & 37 & 32 & 69 & \multirow[t]{2}{*}{5.91} & \multirow[t]{2}{*}{0.05} \\
\hline$\geq 50$ & 47 & 19 & 66 & & \\
\hline \multicolumn{6}{|l|}{ Education } \\
\hline No formal schooling & 40 & 11 & 51 & \multirow[t]{7}{*}{14.91} & \multirow[t]{7}{*}{0.02} \\
\hline Primary schooling not completed & 7 & 4 & 11 & & \\
\hline Primary schooling completed & 2 & 2 & 4 & & \\
\hline Secondary schooling completed & 37 & 18 & 55 & & \\
\hline High school completed & 8 & 7 & 15 & & \\
\hline College completed & 8 & 9 & 17 & & \\
\hline Post graduate & 2 & 7 & 9 & & \\
\hline \multicolumn{6}{|l|}{ Occupation } \\
\hline Agricultural & 0 & 2 & 2 & \multirow{8}{*}{104.33} & \multirow{8}{*}{0.000} \\
\hline Govt Job & 2 & 3 & 5 & & \\
\hline Homemaker & 82 & 0 & 82 & & \\
\hline Private Job & 9 & 15 & 24 & & \\
\hline Retired & 0 & 17 & 17 & & \\
\hline Self employed & 10 & 18 & 28 & & \\
\hline Student & 0 & 3 & 3 & & \\
\hline Unemployed & 1 & 1 & 2 & & \\
\hline \multicolumn{6}{|l|}{ Physical Activity Level at home/work } \\
\hline No exercise and/or sedentary activities & 6 & 5 & 11 & \multirow{4}{*}{4.11} & \multirow{4}{*}{0.12} \\
\hline Regular mild exercise or physical activity & 77 & 34 & 111 & & \\
\hline Regular moderate exercise or manual activity & 21 & 19 & 40 & & \\
\hline $\begin{array}{l}\text { Regular vigorous exercise or strenuous } \\
\text { manual activity }\end{array}$ & 0 & 1 & 1 & & \\
\hline \multicolumn{6}{|l|}{ BMI (Asian classification) } \\
\hline Underweight $<18.5$ & 2 & 2 & 4 & \multirow[t]{4}{*}{3.18} & \multirow[t]{4}{*}{0.36} \\
\hline Normal weight $18.5-22.9$ & 19 & 8 & 27 & & \\
\hline Overweight $23-27.4$ & 44 & 32 & 76 & & \\
\hline Obese $>=27.5$ & 39 & 16 & 55 & & \\
\hline \multicolumn{6}{|l|}{ WC (cm) } \\
\hline$<80(\mathrm{~F})<90(\mathrm{M})$ & 16 & 18 & 34 & \multirow[t]{3}{*}{7.28} & \multirow[t]{3}{*}{0.02} \\
\hline 80-89 (F) 90-99 (M) & 39 & 23 & 62 & & \\
\hline$\geq 90(\mathrm{~F}) \geq 100(\mathrm{M})$ & 49 & 17 & 66 & & \\
\hline \multicolumn{6}{|l|}{ BP (JNC 7 Classification) } \\
\hline Normotensive & 34 & 13 & 47 & \multirow[t]{3}{*}{2.02} & \multirow[t]{3}{*}{0.36} \\
\hline Prehypertensive & 43 & 29 & 72 & & \\
\hline Hypertensive & 27 & 16 & 43 & & \\
\hline \multicolumn{6}{|l|}{ Alcohol user $(n=161)$} \\
\hline Yes & 5 & 12 & 17 & \multirow{2}{*}{10.44} & \multirow{2}{*}{0.005} \\
\hline No & 98 & 46 & 144 & & \\
\hline Eversmoker $(n=161)$ & & & & & \\
\hline Yes & 1 & 8 & 9 & 1215 & 0.002 \\
\hline No & 102 & 50 & 152 & 12.15 & 0.002 \\
\hline
\end{tabular}


Table 2: Mean values of age, BMI and WC among the study participants $(n=162)$ categorized by gender

\begin{tabular}{|lccccc} 
Variable & $\begin{array}{c}\text { Overall Mean } \\
(\mathbf{n}=162)\end{array}$ & $\begin{array}{c}\text { Female }(\mathbf{n}=104) \\
\text { Mean (SD) }\end{array}$ & Male (n=58) & Mean Difference & $\begin{array}{c}\text { Independent t } \\
\text { test P value }\end{array}$ \\
Age (years) & $49.15( \pm 15.12)$ & $47.27( \pm 14.49)$ & $52.51( \pm 15.76)$ & $5.23(0.39-10.07)$ & 0.03 \\
BMI (kg/m2) & $26.10( \pm 4.08)$ & $26.43( \pm 4.52)$ & $25.61 \pm 3.41)$ & $0.82(0.52-2.17)$ & 0.22 \\
WC (cm) & $91.09( \pm 11.26)$ & $89.59( \pm 10.85)$ & $93.79( \pm 11.58)$ & $4.20(0.60-7.80)$ & 0.02 \\
\hline
\end{tabular}

\section{Table 3: Median values with Interquartile range (IQR) of plasma and capillary glucose levels} among the study participants $(n=162)$

\begin{tabular}{|lccccc|}
\hline & \multicolumn{2}{c}{ Overall $(\mathrm{n}=162)$} & Female $(\mathrm{n}=104)$ & Male (n=58) & $\begin{array}{c}\text { Mann Whitney } \\
\text { Variable }\end{array}$ \\
& Mean & Median (IQR) & Median (IQR) & Median (IQR) & (womple test \\
FCG (mg/dl) & 106.47 & $101(95-110)$ & $100(93.5-111)$ & $104(97-110)$ & $1.73, \mathrm{df} 1, \mathrm{p}=0.18$ \\
FPG (mg/dl) & 82.15 & $77(71-85))$ & $75(70-85.5)$ & $79(72-85)$ & $1.14, \mathrm{df} 1, \mathrm{p}=0.28$ \\
$2 \mathrm{hr} \mathrm{PG} \mathrm{(mg/dl)}$ & 94.50 & $81.50(64-107)$ & $80(64-101)$ & $85(64-115)$ & $0.60, \mathrm{df} 1, \mathrm{p}=0.43$ \\
\hline
\end{tabular}

(FCG Fasting capillary blood glucose, FPG Fasting plasma glucose, 2 hr PG 2-hour plasma glucose)

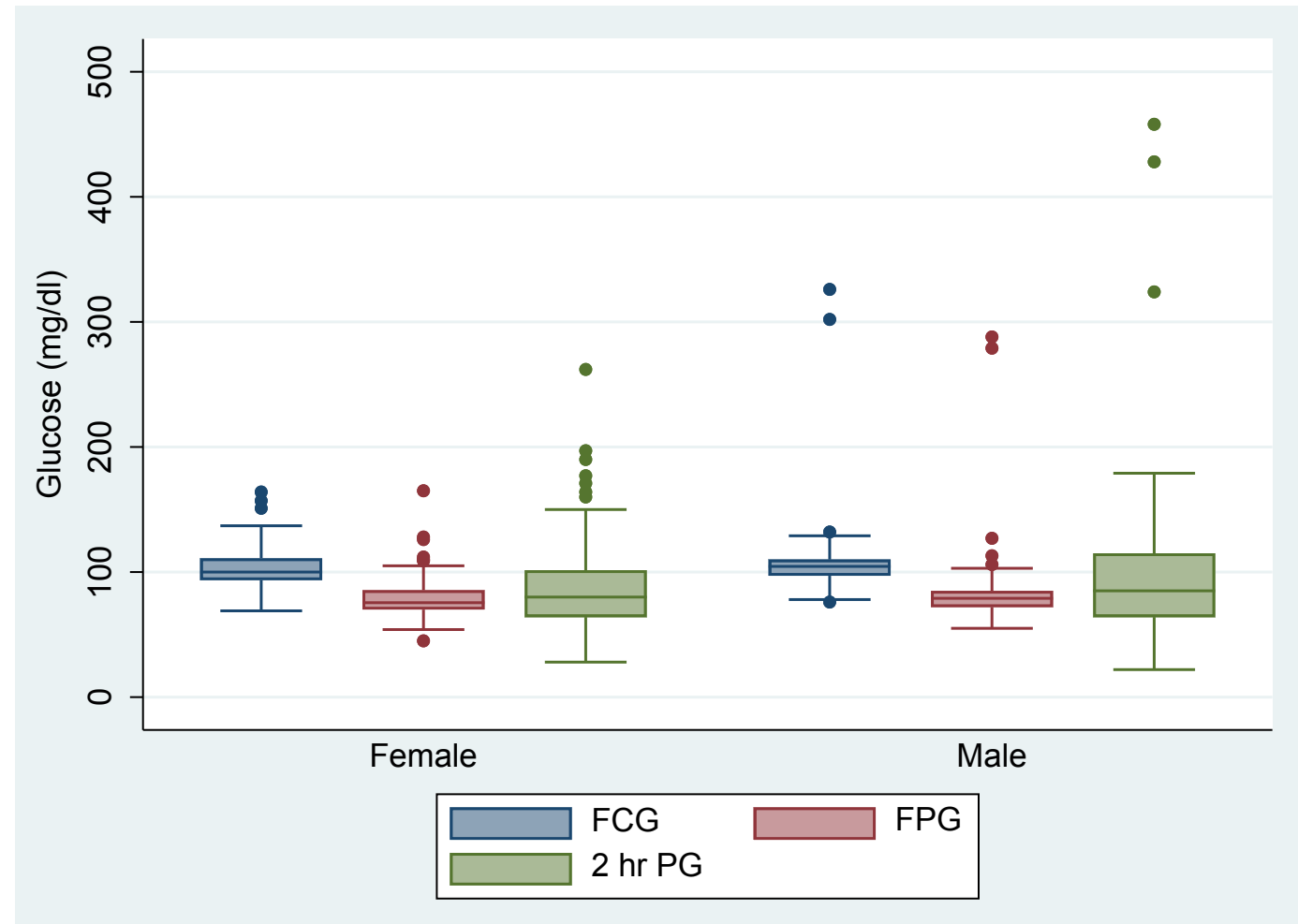

Fig 1: Boxplot of FCG, FPG and 2 hr PG values categorized by gender 


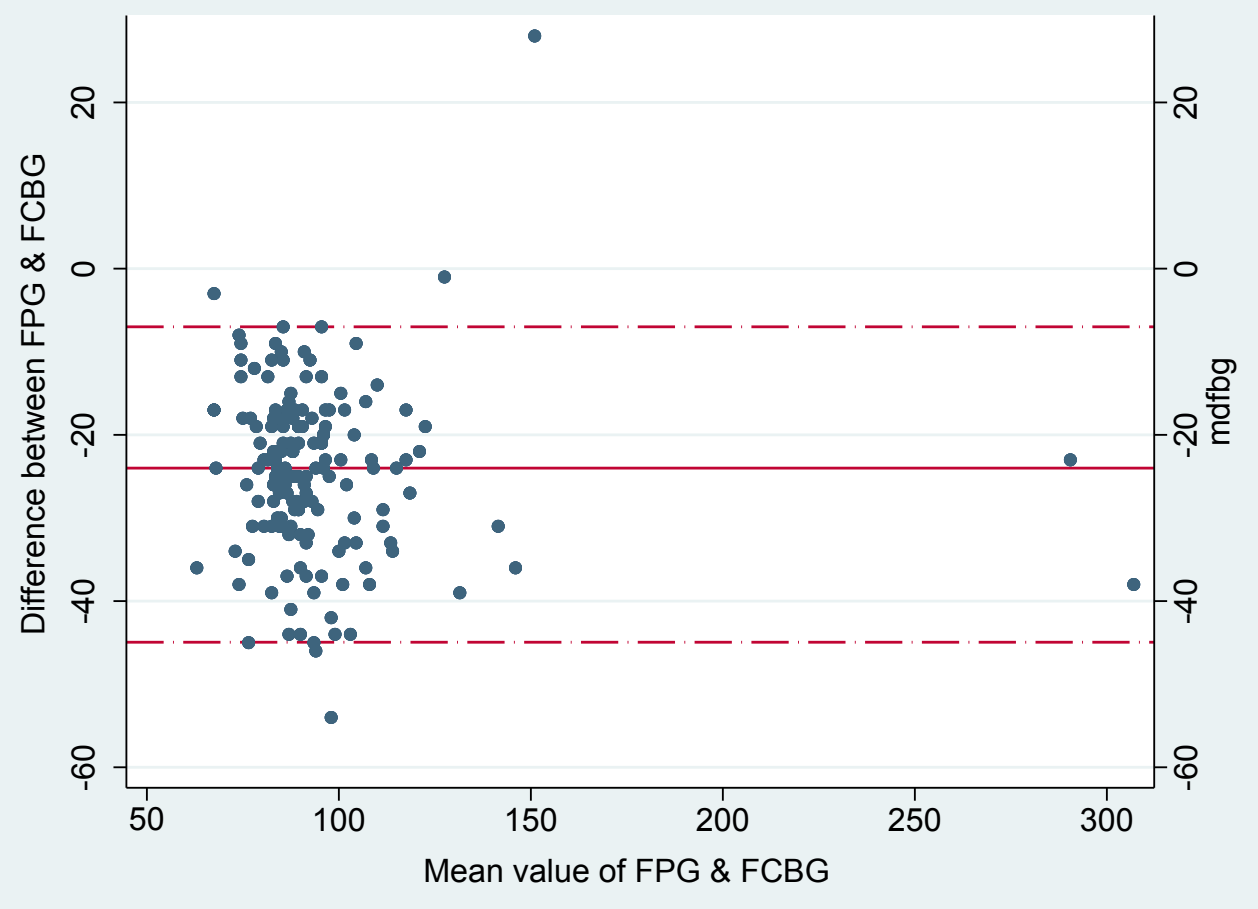

Fig 2: Bland Altman Plot to assess concordance between FCG and FPG measurements

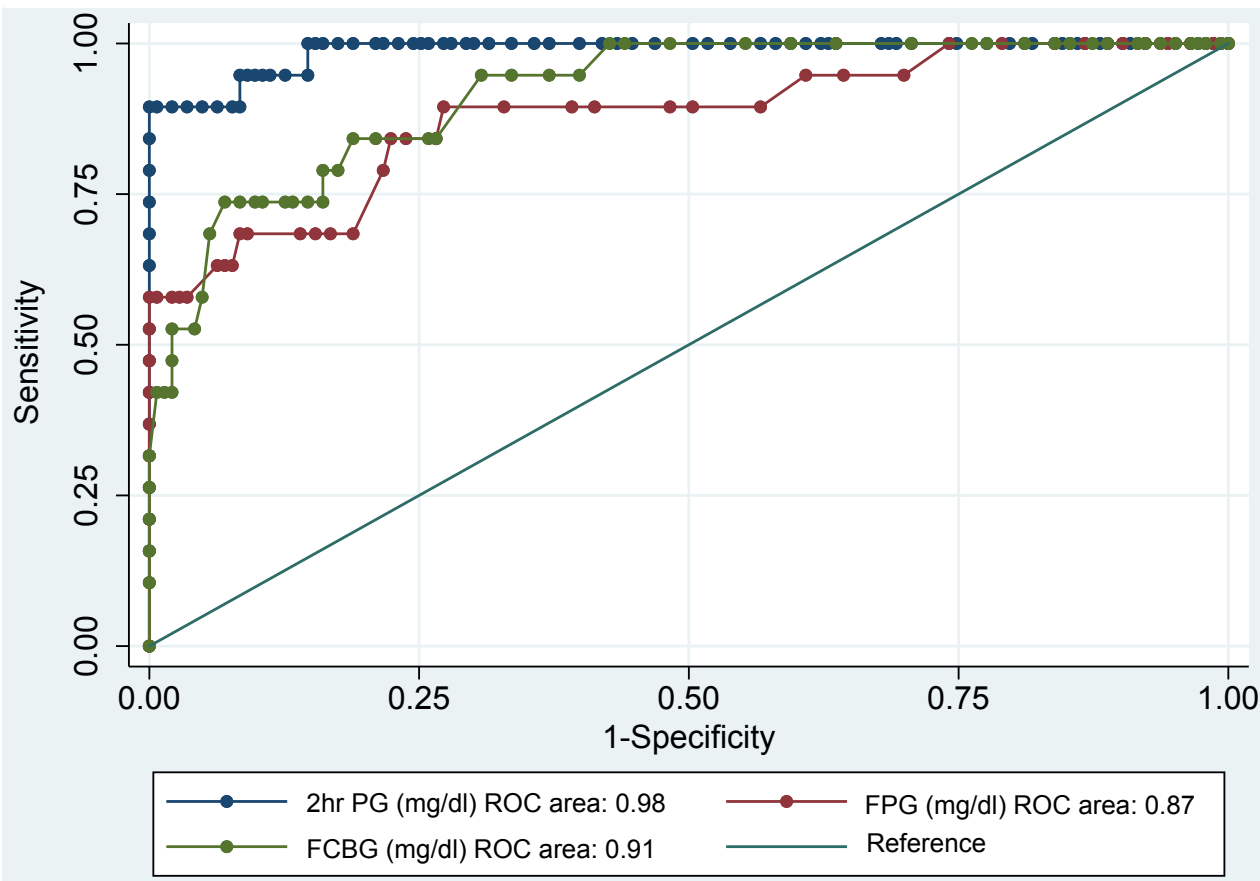

Fig 3: ROC area curve of FCG and FPG against gold standard 2-hour plasma glucose for the identification of diabetes and prediabetes

\section{Table 4: Prevalence of undiagnosed diabetes and prediabetes categorized by gender (WHO} 1999 classification)

\begin{tabular}{lccccc} 
WHO Diabetes classification & Female & Male & TOTAL & Chi-square value & P value \\
Diabetes & 4 & 3 & 7 & & \\
Prediabetes (IGT) & 6 & 6 & $\mathbf{1 2}$ & $\mathbf{1 . 3 5}$ & $\mathbf{0 . 5}$ \\
Normoglycaemia & 94 & 49 & $\mathbf{1 4 3}$ & & \\
TOTAL & $\mathbf{1 0 4}$ & $\mathbf{5 8}$ & $\mathbf{1 6 2}$ & & \\
\hline
\end{tabular}


Table 5: Correlation between FCG, FPG and 2 hr PG measurements

\begin{tabular}{lccccc|} 
Variable & FPG $(\boldsymbol{\beta})$ & $\mathbf{9 5 \%}$ CI $\boldsymbol{\beta}$ & $\mathbf{r}$ & $\mathbf{r}^{2}$ & P value \\
FCG & 0.90 & 0.85 to 0.96 & 0.67 & 0.86 & $<0.0001$ \\
& 2 hr PG $(\beta)$ & & & & \\
FCG & 1.60 & 1.39 to 1.80 & 0.45 & 0.60 & $<0.0001$ \\
FPG & 1.69 & 1.49 to 1.89 & 0.49 & 0.64 & $<0.0001$ \\
\hline
\end{tabular}

(FCG Fasting capillary glucose, FPG Fasting plasma glucose, 2 hr PG 2-hour plasma glucose, Beta coefficient $\beta, 95 \%$ CI $\beta$ Confidence intervals for beta coefficient, $r$ Spearman's correlation coefficient, $r^{2}$ Coefficient of determination)

\begin{tabular}{|lccccc|}
\multicolumn{5}{|c|}{ Table 6: ROC area curve of FCG, FPG and 2-hour plasma glucose with 95\% CI for the } \\
identification of diabetes and prediabetes \\
\hline Variable & Number & ROC Area & SE & 95\% CI & ROC AUC Equality Test P value \\
FCG & 162 & 0.91 & 0.03 & 0.85 to 0.97 & 0.40 \\
FPG & 162 & 0.87 & 0.04 & 0.78 to 0.97 & 0.04 \\
2 hr PG & 162 & 0.98 & 0.009 & 0.97 to 1.00 & \\
\hline
\end{tabular}

Table 7: Receiver Operator Characteristic Curve Sensitivity and Specificity values for FCG and FPG cut-points

\begin{tabular}{lccccc|}
$\begin{array}{l}\text { Glycaemic cut } \\
\text { point (mg/dl) }\end{array}$ & $\begin{array}{c}\text { Sensitivity } \\
\text { (\%) }\end{array}$ & $\begin{array}{c}\text { Specificity } \\
\text { (\%) }\end{array}$ & $\begin{array}{c}\text { Correctly } \\
\text { Classified (\%) }\end{array}$ & $\begin{array}{c}\text { Positive } \\
\text { LR }\end{array}$ & $\begin{array}{c}\text { Negative } \\
\text { LR }\end{array}$ \\
FCG & & & & & \\
$\geq 100$ & 100 & 51.75 & 57.41 & 2.07 & 0.00 \\
$\geq 110$ & 84.21 & 81.12 & 81.48 & 4.46 & 0.19 \\
$\geq 126$ & 57.89 & 95.10 & 90.74 & 11.82 & 0.44 \\
FPG & & & & & \\
$\geq 100$ & 57.89 & 97.20 & 92.59 & 20.69 & 0.43 \\
$\geq 110$ & 47.37 & 100 & 93.83 & & 0.52 \\
$\geq 126$ & 31.58 & 100 & 91.98 & & 0.68 \\
\hline
\end{tabular}

On ROC curve analysis of FCG cut-offs for the identification of persons with 'raised blood glucose', the cut-off level of $110 \mathrm{mg} / \mathrm{dl}$ and above had an optimal sensitivity and specificity of $84.21 \%$ and $81.12 \%$ respectively. The accuracy was $81.48 \%$ with a positive likelihood ratio of 4.46 and the negative likelihood ratio of 0.19 at this cut-off. In comparison, at $110 \mathrm{mg} / \mathrm{dl}$, the sensitivity and specificity of FPG was $47.37 \%$ and $100 \%$. At a cut-off value of $100 \mathrm{mg} / \mathrm{dl}$, the sensitivity and specificity of FCG was $100 \%$ and $51.75 \%$ in comparison to $57.89 \%$ and $97.20 \%$ for FPG, (Table 7).

\section{DISCUSSION}

Undiagnosed diabetes and prediabetes appear to be a significant morbidity in this semi-urban community. Though low educational level and abdominal obesity was more prevalent among the women, an association between gender and glycaemic status was not evident. However, a systematic review in Nepal has suggested that women may be at a higher risk of diabetes due to their low educational status. There appears to be a need for gender specific studies in diabetes in Nepal. ${ }^{14}$

The difference between FCG and FPG values suggested analytical inaccuracy of the glucose meter according to the ISO 15197:2013 guideline. Statistical tools which measure accuracy have a limited meaning to the clinician and persons with diabetes who need to know how close the meter reading is to the true plasma glucose value. Errors can arise during the use of glucose meters, however FCG measurements were found to be 
clinically accurate as demonstrated by the Clarke error grid in a separate analyses carried out by the investigators..$^{21,22}$

The concordance between FCG and FPG measurements was found to be acceptable with $97 \%$ of the readings lying within the limits of agreement on the BA plot. The concordance was further supported by the large correlation coefficient between FCG and FPG measurements. At each recommended cut-off value, FCG was found to be more sensitive than FPG in the identification of persons with RBG. From the public health perspective, FCG may be more suitable for screening in comparison to the more specific and accurate FPG. The optimal cut-off for FCG was $110 \mathrm{mg} / \mathrm{dl}$ for screening for both diabetes and prediabetes. If FPG were to be used as a screening tool, then a lower cut-off of $100 \mathrm{mg} / \mathrm{dl}$ may be more suitable than the WHO recommended cutoff of $110 \mathrm{mg} / \mathrm{dl}$ as the sensitivity increased with the lower value.

Within 95\% confidence intervals, FCG was able to identify between $85 \%$ to $97 \%$ of the persons with raised blood glucose in comparison to $78 \%$ to $97 \%$ for FPG. As there was no significant difference in the ROC AUC of the two screening tests, FCG may be a convenient and cost-effective tool for detection of prediabetes and diabetes in community based and primary health care settings.

In comparison to the present study findings, a study among 993 adults in the Yunnan province of China reported a higher prevalence of prediabetes and diabetes $(14.60 \%$ \& $5.75 \%)$ and a lower optimal FCG cut-off for screening. Concordance between FCG and FPG measurements was high with a Pearson correlation coefficient of 0.75 $(p<0.0001)$. The ROC area under the curve for FCG was 0.88 (95\% CI 0.82 to 0.93 ) in comparison to 0.92 (95\% CI 0.88 to 0.97) for FPG. The optimal cut-off for FCG was found to be $101 \mathrm{mg} / \mathrm{dl}$ with a sensitivity and specificity of $84.2 \%$ and $79.3 \%$. The investigators concluded that there was no significant difference in the performance of FCG and FPG. FCG was deemed to be a convenient and practical screening strategy in low resource settings which concur with the present study findings. ${ }^{23}$

Utilizing data from the Qingdao Diabetes prevention study in China, the performance of FCG, FPG, 2 hr PG, HbA1c and a diabetes risk score was evaluated among 4070 persons. The sensitivity, specificity and ROC AUC for FCG was $64.08 \%, 69.81 \%$ and 0.71 in comparison to $60.10 \%, 100 \%$ and 0.89 for FPG. For the DRS, it was $61.50 \%, 63.72 \%$ and 0.67 respectively. Though the sensitivity and specificity of FCG in this large study was lower in comparison to other studies,
FCG and DRS were deemed to be suitable tools for screening. ${ }^{24}$

In another study among 2332 adults in Qingdao, China, the performance of FCG and glycated $\mathrm{Hb}$ was evaluated using 75g OGTT. The prevalence rates of undiagnosed diabetes and prediabetes $(11.9 \%$ \& $29.5 \%)$ were higher indicating greater disease burden. For the identification of persons with diabetes, the ROC AUC was 0.77 for FCG and 0.67 for HbA1c for men. For women, it was 0.75 and 0.67. For the identification of prediabetes, the ROC AUC was 0.64 for FCG and 0.47 for HbA1c for men. For women, it was 0.65 and 0.51. FCG performance was found to be better than HbA1c as a screening tool. ${ }^{25}$

However, a study carried out among 669 persons from a rural area of Thailand for the screening of T2D alone reported a sensitivity of less than $50 \%$ with specificity of over $96 \%$ for FCG and FPG at a cut-off of $126 \mathrm{mg} / \mathrm{dl}$ in comparison to the reference HbA1c. ${ }^{26}$

In 1998, the Diabetes Epidemiology Collaborative analysis of Diagnostic criteria in Europe (DECODE) study had suggested that FPG tended to underestimate the prevalence rate of diabetes and prediabetes. The cut-off for IFG in the DECODE study was $110 \mathrm{mg} / \mathrm{dl} .{ }^{27}$

The Diabetes mellitus and vascular health initiative, a large screening study in Ireland also reported that FPG as an initial screening test would lead to an underestimation of undiagnosed diabetes. Among 29,144 adults, the prevalence of undiagnosed T2D, IFG and IGT was reported as $1.8 \%, 7.1 \%$ and $2.9 \%$ respectively. The study adopted the ADA recommended cut-off of 100 $\mathrm{mg} / \mathrm{dl}$ for IFG. Lowering the cut-off increased the sensitivity of the FPG which was concurrent with the present study findings. ${ }^{28}$

The evaluation of the IDRS in the present study population suggested that it may be a suitable non-invasive screening tool. The cut-off score of 60 and above was found to have a sensitivity and specificity of $84.21 \%$ and $55.24 \%$ with an accuracy of $58.64 \%$. Within $95 \%$ confidence intervals, IDRS was able to identify between $62 \%$ to $77 \%$ of the persons with raised blood glucose in comparison to $78 \%$ to $97 \%$ for FPG. $^{16}$ Combining the IDRS with FCG estimation may increase the sensitivity and increase the yield during community based screening. ${ }^{29}$

Amongst the study participants, a larger proportion of prediabetes was seen in comparison to undiagnosed diabetes. Previous economic evaluations of opportunistic screening tests have suggested that screening for both the conditions (diabetes and prediabetes) may be cost saving 
from a health perspective. From a societal perspective, a similar screening may be cost neutral. The evaluation found that the cost per case for diabetes and prediabetes screening was lower as compared to screening for diabetes alone. The sensitivity of combined screening was estimated to range from $67-82 \%$. Hence, screening for both diabetes and prediabetes should be considered along with measures for management. ${ }^{29,30}$

A majority of primary prevention studies have been carried out in persons with prediabetes (IGT) across diverse populations. The American Diabetes Prevention Program which was the largest trial for lifestyle interventions included a minimum of $7.0 \%$ weight loss, decreased consumption of calories and dietary fat along with 150 minutes of physical activity in a week. The number needed to treat to prevent 1 case of diabetes was seven for lifestyle interventions and fourteen for Metformin. The risk reduction with lifestyle interventions was significant across all age, ethnicity and BMI categories. There is level 1 evidence that lifestyle interventions can reduce the incidence of diabetes or delay the progression from a state of impaired glucose tolerance to frank diabetes by $50-60 \% .^{31-34}$

In our study setting, we suggest that communitybased screening for prediabetes and diabetes may be carried out by risk assessment with of IDRS and estimation of FCG. Persons with an IDRS score of 60 and above or with FCG level of
$110 \mathrm{mg} / \mathrm{dl}$ and above can be advised to undergo an OGTT with further cardiovascular assessment and management.

Strengths of the study: Recognizing the use of glucometer and capillary sampling in low- and middle-income countries, the study was planned to assess the performance of FCG in a 'real world setting'. The glucometer was calibrated to report plasma concentrations and OGTT testing was carried out for definitive testing following WHO/ IDF guidelines. ${ }^{1}$

Limitations: The venous blood samples were collected in a container with glycolytic inhibitors however, plasma separation and laboratory analysis were performed within 4 hours after collection. Though two glucometers were used during the screening camp, within glucometer variation was not studied.

In conclusion, at each recommended cut-off value, FCG was found to be more sensitive than FPG for the identification of persons with diabetes and prediabetes. As there was no significant difference between ROC AUC of the two tests, FCG may be a suitable, sensitive and convenient screening tool in community-based settings in comparison to the more specific and accurate FPG. Larger prospective studies may help to further elucidate the cost-effectiveness and efficacy of a similar screening strategy in the Nepalese community.

\section{REFERENCES}

1. WHO. Definition and diagnosis of diabetes mellitus and intermediate hyperglycaemia. Geneva: World Health Organization Press. Epub ahead of print 2006. DOI: ISBN 9241594934.

2. Porta M, Curletto G, Cipullo D et al. Estimating the delay between onset and diagnosis of type 2 diabetes from the time course of retinopathy prevalence. Diabetes Care 2014; 37: 1668-74.

3. Harris MI, Klein R, Welborn TA et al. Onset of NIDDM occurs at least 4-7 yr before clinical diagnosis. Diabetes Care 1992; 15: 815-9.

4. Heuck C-C, Home PD, Reinauer H et al. Laboratory Diagnosis and Monitoring of Diabetes Mellitus. World Heal Organ 2002; 1-26.

5. Beagley J, Guariguata L, Weil C et al. Global estimates of undiagnosed diabetes in adults. Diabetes Res Clin Pract; 103. Epub ahead of print 2014. DOI: 10.1016/j.diabres.2013.11.001.

6. WHO. WHO (1999) Definition, Diagnosis and Classification of Diabetes Mellitus. 1999; 1-59.

7. American Diabetes Association. Standards of Medical care in diabetes - 2017. J Clin Appl Res Educ 2017; 40: 1-142.
8. WHO. Global Report on Diabetes. 2016; 88.

9. Abbasi A, Peelen LM, Corpeleijn E et al. Prediction models for risk of developing type 2 diabetes: systematic literature search and independent external validation study. Brit Med J 2012; 345. Available at: http://www.bmj.com/content/345/bmj. e5900.abstract (2012).

10. Somannavar S, Ganesan A, Deepa M et al. Random capillary blood glucose cut points for diabetes and pre-diabetes derived from community-based opportunistic screening in India. Diabetes Care 2009; 32: 641-3.

11. Freckmann G, Schmid C, Baumstark A et al. System accuracy evaluation of 43 blood glucose monitoring systems for self-monitoring of blood glucose according to DIN EN ISO 15197. J Diabetes Sci Technol 2012; 6: 1060-75.

12. Ginsberg BH. Factors Affecting Blood Glucose Monitoring: Sources of Errors in Measurement. $J$ diabetes Sci Technol 2009; 3: 903-13.

13. Klonoff DC, Prahalad P. Performance of Cleared Blood Glucose Monitors. J Diabetes Sci Technol 2015; 9: 895-910. 
14. Gyawali B, Sharma R, Neupane D et al. Prevalence of type 2 diabetes in Nepal: a systematic review and meta-analysis from 2000 to 2014. Global Health Action 2015; 8: 10.3402/gha.v8.29088.

15. Mohan V, Deepa R, Deepa $\mathrm{M}$ et al. A simplified Indian Diabetes Risk Score for screening for undiagnosed diabetic subjects. J Assoc Physicians India 2005; 53: 759-63.

16. Silvanus V, Dhakal N, Pokhrel A, Baral BK, Panta P. Community based screening for diabetes and prediabetes using the Indian Diabetes Risk Score among adults in a semi-urban area in Kathmandu, Nepal. Nepal Med Coll J 2019; 21: 12-20.

17. Khunti K, Mani H, Achana F et al. Systematic Review and Meta-Analysis of Response Rates and Diagnostic Yield of Screening for Type 2 Diabetes and Those at High Risk of Diabetes. PLOS One 2015; 10: e0135702.

18. WHO. The WHO STEPwise approach to noncommunicable disease risk factor surveillance. World Heal Organ 2017; 1-474.

19. IDF Guideline Development Group. Global guideline for type 2 diabetes. Diabetes Res Clin Pract 2014; 104: 1-52.

20. Kwiecien R, Kopp-Schneider A, Blettner M. Concordance analysis: part 16 of a series on evaluation of scientific publications. Dtsch Arztebl Int'l 2011; 108: 515-21.

21. Pokhrel A, Silvanus V, Pokhrel BR et al. Accuracy of Glucose Meter among Adults in a Semi-urban Area in Kathmandu, Nepal. J Nepal Med Assoc 2019; 57: 104-8.

22. Boren SA, Clarke WL. Analytical and clinical performance of blood glucose monitors. J Diabetes Sci Technol 2010; 4: 84-97.

23. Zhao $\mathrm{X}$, Zhao $\mathrm{W}$, Zhang $\mathrm{H}$ et al. Fasting capillary blood glucose: an appropriate measurement in screening for diabetes and pre-diabetes in lowresource rural settings. J Endocrinol Invest 2013; 36: 33-7.

24. Wang X, Li S, Sun X, Pang Z, Qiao Q. Evaluation of effects of five screening methods for asymptomatic type 2 diabetes. J Shandong Univ (Health Sci) 2011; 49: 158-62.

25. Zhou X, Pang Z, Gao W et al. Performance of an $\mathrm{A} 1 \mathrm{C}$ and fasting capillary blood glucose test for screening newly diagnosed diabetes and prediabetes defined by an oral glucose tolerance test in Qingdao, China. Diabetes Care 2010; 33: 545-50.

26. Muktabhant B, Sanchaisuriya P, Sarakarn P et al. Use of glucometer and fasting blood glucose as screening tools for diabetes mellitus type 2 and glycated haemoglobin as clinical reference in rural community primary care settings of a middle income country. BMC Public Health 2012; 12: 349.

27. Glucose tolerance and mortality: comparison of WHO and American Diabetes Association diagnostic criteria. The DECODE study group. European Diabetes Epidemiology Group. Diabetes Epidemiology: Collaborative analysis Of Diagnostic criteria in Europe. Lancet (London, England) 1999; 354: 617-21.

28. Sinnott M, Kinsley BT, Jackson $\mathrm{AD}$ et al. Fasting plasma glucose as initial screening for diabetes and prediabetes in irish adults: The Diabetes Mellitus and Vascular health initiative (DMVhi). PLoS One 2015; 10: e0122704.

29. Khunti K, Gillies CL, Taub NA et al. A comparison of cost per case detected of screening strategies for Type 2 diabetes and impaired glucose regulation: modelling study. Diabetes Res Clin Pract 2012; 97: 505-13.

30. DECODE Study Group. Will new diagnostic criteria for diabetes mellitus change phenotype of patients with diabetes? Reanalysis of European epidemiological data. DECODE Study Group on behalf of the European Diabetes Epidemiology Study Group. Brit Med J 1998; 317: 371-5.

31. Torjesen PA, Birkeland KI, Anderssen SA et al. Lifestyle Changes May Reverse Development of the Insulin Resistance Syndrome. The Oslo Diet and Exercise Study: a randomized trial. Diabetes Care 1997; 20: 26-31.

32. Pan XR, Li GW, Hu YH et al. Effects of diet and exercise in preventing NIDDM in people with impaired glucose tolerance. The Da Qing IGT and Diabetes Study. Diabetes Care 1997; 20: 537-44.

33. Lindstrom J, Louheranta A, Mannelin M et al. The Finnish Diabetes Prevention Study (DPS). Diabetes Care 2003; 26: 3230-6.

34. DPP Research Group. The Diabetes Prevention Program (DPP): Description of lifestyle intervention. Diabetes Care 2002; 25: 2165-71. 